\title{
Opportunity for change: is it time to redefine the role of paramedics in healthcare?
}

\author{
Ian R. Drennan ${ }^{1} \cdot$ Ian E. Blanchard ${ }^{2,3} \cdot$ Jason E. Buick ${ }^{4}$
}

Received: 14 January 2021 / Accepted: 9 February 2021

(c) The Author(s), under exclusive licence to Canadian Association of Emergency Physicians (CAEP)/ Association Canadienne de Médecine d'Urgence (ACMU) 2021

Paramedics are often the first point of contact for patients who access emergency healthcare services and are faced with a myriad of situations from low acuity complaints to life-threatening emergencies. Paramedics have been at the forefront of the COVID-19 pandemic, responding to emergencies, often in uncontrolled, unpredictable, and austere environments.

During the pandemic, many paramedic services have reported significant changes to service utilization $[1,2]$. In this issue of CJEM, Grunau and colleagues highlight some of these changes, the challenges in pandemic response planning, and the role that paramedics play in helping to care for patients. Grunau et al. identified a $15 \%$ decrease in overall call volume during the first few months of the pandemic, and a 9\% decrease in calls for major trauma, ST-segment elevation myocardial infarction (STEMI), and stroke [3]. They also identified changes in the primary problem for patients who called 911. There was a higher incidence of calls for respiratory distress and anxiety and a decrease in all other primary complaints. Interestingly, the authors found no change in the incidence of cardiac arrest calls [3].

There are a multitude of potential explanations for the noted decrease in 911 calls. First, the decrease in calls could be due to changes in patient behaviour, perhaps motivated by fear of contracting COVID-19. It is conceivable that patients

Ian R. Drennan

Ian.Drennan@Sunnybrook.ca

1 Sunnybrook Research Institute, Sunnybrook Health Sciences Centre, 2075 Bayview Avenue, Toronto, ON M4N 3M5, Canada

2 Emergency Medical Services, Alberta Health Services, AB, Canada

3 Department of Community Health Sciences, Cumming School of Medicine, University of Calgary, Calgary, AB, Canada

4 Institute of Health Policy Management and Evaluation, University of Toronto, Toronto, ON, Canada (and their families) viewed hospitals as high-risk locations for COVID-19 transmission, which led to delayed or absent 911 calls; even for people with time-sensitive life-threatening conditions. This has been reported by others who noted reductions in STEMI and stroke cases during the pandemic $[4,5]$. Another explanation may be the general public heeding public health advice to only use the healthcare system if absolutely necessary to prevent overburdening the system, resulting in a hesitation to call for help even when it may be required.

Second, the reduction in call volumes could be a result of pandemic induced restrictions, resulting in fewer personto-person interactions, and fewer people moving about each day in the community. While this could explain changes in certain conditions such as traumatic injuries, it is difficult to know whether this can explain other conditions such as heart attacks and strokes, which are the result of chronic disease processes. The reduction in call volume is likely related to a unique constellation of healthcare system issues including social, geographic, public health, and paramedic factors that are location specific. This may explain the contradiction in findings to other services that have reported increases in paramedic call volume [6], especially in cardiac arrests during the same time period $[2,6]$.

Lastly, it is possible the pandemic has exposed a larger issue; the over-utilization of 911 by patients with non-urgent complaints. While not explicitly the focus of the paper by Grunau and colleagues, it is well documented elsewhere, that an increasing proportion of 911 patients have non-urgent complaints and many are not transported to an emergency department (ED) [7]. Patients who have poor access to healthcare services often feel as if they have no alternatives and call 911 to access medical care. Other patients are unsure what constitutes a true medical emergency and are looking for assessment and advice. Either way these patients are not necessarily looking for transport to the ED. Perhaps this pandemic allows for an opportunity to re-examine the 
role of paramedics within the healthcare system to optimize patient care and system efficiency.

Paramedics have traditionally provided on-scene assessments and stabilization prior to transport to the ED for further care. There is, however, increasing awareness that this "you call, we haul" model is not financially sustainable, contributes to ED overcrowding, and does not result in a patient-centred approach to care [8]. Current models of care need to adapt to meet the growing and changing demand for community-based service. Unfortunately, the often antiquated system under which many paramedics work has not evolved to meet this need. This has become more apparent during the pandemic, where the rate of non-transport to hospital in many systems has substantially increased [1]. Paramedics are more often being asked to perform assessments and provide patient care on-scene without transporting patients to the ED, thereby performing a critical triaging function within the healthcare system. In Ontario, for instance, a COVID-19 directive has been developed to allow paramedics to initiate sign offs for low-risk COVID patients; reducing the transport burden on the system. These tools improve efficiencies within the system while ensuring safe and appropriate patient care.

While we are still in the middle of a global pandemic, we should continue to look to the future for ways to optimize delivery of out-of-hospital care. The work by Grunau and colleagues presents a starting point for this discussion, and a unique opportunity to continue to evaluate patient perceptions, attitudes, and barriers to accessing emergency services during the pandemic. In addition, it is important to evaluate additional assessments, treatments and referrals paramedics could provide at the scene for patients who do not want, or do not require transport to the ED.

This proactive approach could help us in future times of crisis. In addition, thorough evaluation of 911 use across Canada provides a vital opportunity to re-evaluate the system as a whole, including the role of paramedics within the larger healthcare system. Development of protocols and decision-making support ensures patient-centred care by allowing paramedics to assess, treat, and refer patients to other health resources at the scene avoiding unnecessary exposure of patients and ED staff, and helping to alleviate excess burden on beleaguered EDs. Re-development of the role of paramedics in other countries has demonstrated positive results from both a patient and system perspective [9].
Many questions remain surrounding the role of paramedics. Will we continue to see changes in 911 calls in subsequent waves of the pandemic? Have other jurisdictions in Canada had a similar experience? And how can we support paramedics to provide safe and appropriate patient-centred care in the community? Grunau and colleagues provide us with a valuable starting point for discussion on the role that paramedics play in healthcare in Canada, and an exemplar of what can be achieved with paramedic and physician collaboration.

\section{References}

1. Lane DJ, Blanchard IE, Buick JE, et al. Contrasting the impact of the COVID-19 pandemic on Emergency Medical Services and Emergency Department frequency, presenting severity, and disposition. Canadian Medical Association Journal Open. 2021. (In Press)

2. Lerner EB, Newgard CD, Mann NC. Effect of the coronavirus disease 2019 (COVID-19) pandemic on the U.S. emergency medical services system: a preliminary report. AcadEmerg Med. 2020;27(8):693-9.

3. Grunau B, Helmer J, Lee S, et al. Decrease in Emergency Medical Services Utilization During Early Stages of the COVID-19 Pandemic in British Columbia. Canadian Journal of Emergency Medicine. 2021. https://doi.org/10.1007/s43678-020-00062-y.

4. Baracchini C, Pieroni A, Viaro F, et al. Acute stroke management pathway during Coronavirus-19 pandemic. Neurologic Science. 2020;41(5):1003-5.

5. Baum A, Schwartz MD. Admissions to veterans affairs hospitals for emergency conditions during the COVID-19 pandemic. JAMA. 2020;324(1):96-9.

6. Prezant DJ, Lancet EA, Zeig-Owens R, Lai PH, Appel D, Webber MP, et al. System impacts of the COVID-19 pandemic on New York City's emergency medical services. J Am CollEmerg Physicians Open. 2020;1:1205-13.

7. Eastwood K, Morgans A, Smith K, Hodgkinson A, Becker G, Stoelwinder J. A novel approach for managing the growing demand for ambulance services by low-acuity patients. Australian Health Rev. 2016;40:378-84.

8. Office of the Auditor General of Ontario. 2013 Annual Report. Chapter 3. Section 3.04: Land Ambulance. 2013. URL: https ://www.auditor.on.ca/en/content/annualreports/arbyyear/ar201 3.html Accessed: January 52021.

9. Blodgett JM, Robertson DJ, Pennington E, Ratcliffe D, Rockwood K. Alternatives to direct emergency department conveyance of ambulance patients: a scoping review of the evidence. Scand J Trauma Resusc Emerg Med. 2021;29(4):1-21. 